\title{
Control of microbial contamination in unpreserved eyedrops
}

\author{
Gillian B Oldham, Victor Andrews
}

\begin{abstract}
Aims/background-Preservatives are added to multidose eyedrop containers to ensure that the eyedrops do not become heavily contaminated during patient use. This enables eyedrops to be used for up to 1 month after opening. However, patients are frequently required to use unpreserved eyedrops as their eyes are unable to tolerate these preservatives. Some commercial unpreserved unit dose eyedrops are available, but the range is limited, and they cannot be used in all cases. Twenty one different unpreserved eyedrop formulations in multidose bottles were tested to establish their inherent efficacy in antimicrobial preservation, and to help determine a suitable in use storage life.

Methods-The eyedrops were inoculated with a known quantity of four different micro-organisms according to the method of the European Pharmacopoeia 'Test for the efficacy of antimicrobial preservation'. After set periods of time, samples were taken to determine the number of viable organisms remaining.

Results-Antibiotics and alkaloids were generally shown to have higher kill rates than other eyedrops such as artificial tears and steroids.

Conclusions-In general, once opened by individual patients in a domiciliary situation, a 7 day in use storage life is confirmed for eyedrops containing alkaloids or antibiotics, if they are stored in the refrigerator after opening.

(Br f Ophthalmol 1996;80:588-591)
\end{abstract}

Aqueous eyedrops supplied in multidose containers include suitable antimicrobial preservatives at appropriate concentrations, except when the preparation itself has adequate antimicrobial properties. These preservatives must be compatible with the other ingredients of the preparation, and must remain effective throughout the period of use of the eyedrops. ${ }^{1}$

Increasingly, it has become apparent that a small but significant number of patients are intolerant of preservatives, ${ }^{2-4}$ either because of ocular toxicity which may be due to the disease process itself, or because of hypersensitivity or allergy. It is for this reason that there is a small but significant clinical need for eyedrops to be prepared without added preservatives.

Unpreserved eyedrops are available in unit dose containers or vials (UDVs), and they are designed primarily for use in surgical situations. However, the practicality and expense of using UDVs in a domiciliary situation must be considered. Patients using drops for frequent administration find UDVs too cumbersome and can have difficulty carrying a sufficient supply for their needs. Also, the cost of using these UDVs can be up to $1169 \%$ more expensive than treating patients with the equivalent preserved eyedrops. $^{5}$

Since the equipment to manufacture UDVs costs upwards of $£ 2$ million, the lack of available equipment in the hospital pharmacy necessitates the manufacture of unpreserved eyedrops in multidose bottles.

The British National Formulary $(B N F)^{6}$ states that eyedrops in multiple application containers for domiciliary use should not be used for more than 4 weeks after opening (unless otherwise justified and authorised). This information was originally published in the British Pharmaceutical Codex ${ }^{7}$ but it appears that this has always been an arbitrary figure as studies have never been conducted to confirm the validity of this opened storage life. The Health Service Circular ${ }^{8}$ addressed the preservation of sterility in ophthalmic preparations in hospitals, but again the recommendations made in this document were never based on scientific data and, in any case, none of these references mentions unpreserved eyedrops in multidose containers. The arbitrary nature of all of these recommendations probably explains why the United States Pharmacopeia (USP) ${ }^{9}$ has not made any attempt to follow suit with recommendations of its own.

The Medicines Act Information Leaflet ${ }^{10}$ gives storage recommendations for reconstituted powders for injections and recommends that when a powder is reconstituted with unpreserved diluent, the storage life of the reconstituted product should not be longer than 24 hours stored at $2-8^{\circ} \mathrm{C}$, in order to reflect good microbiological practice. Many people have extended this as a recommendation for the in use storage life of ophthalmic products. However, a large number of oph- 
thalmic drugs have their own inherent antimicrobial activity and therefore this in use storage life may not always be appropriate.

\section{Methods}

SIGNIFICANCE OF METHODOLOGY

Many papers have been published on the investigation of the levels of bacterial contamination in used eyedrops ${ }^{11-14}$ by recovering the discarded eyedrop bottles from patients, but there is an inevitable delay in analysing samples after patients have finished using them. For instance, test samples may have been mailed at room temperature to the analytical laboratory. While this may not be critical for preserved eyedrops, it may significantly influence the apparent degree of contamination seen with unpreserved eyedrops. Our recommendation for unpreserved drops is to keep them refrigerated at all times once opened, and any subsequent length of time spent at room temperature for unpreserved drops means that sufficient quantities of micro-organisms may grow and be detected during the test procedure. If unpreserved drops were refrigerated at all times, the microbial multiplication rate could be significantly less and might be below the limit of detection. As very low levels of micro-organisms are unlikely to cause a clinical incidence, some degree of quantification is important.

Both the USP ${ }^{9}$ and the European Pharmacopoeia $(E P)^{16}$ give official quantitative methods for testing the efficacy of antimicrobial preservation in ophthalmic solutions. However, there are important differences between these two tests. The most notable and relevant of these differences is the reduction in count of microorganisms. The USP requires a $\log 3$ reduction at 14 days in the case of bacteria, while the EP recommends this same reduction in 24 hours. Also, the USP requires that there is no increase in fungal count throughout the period of the test, whereas the EP requires a reduction in the number of fungi in 7-14 days. While these differences are significant for some preserved eyedrops, ${ }^{11}$ for unpreserved eyedrops the $E P$ test is a more appropriate test as it provides information about micro-organisms in the short term (that is, at 6 and 24 hours), as well as at 7 days. For this reason, the $E P$ test for the efficacy of antimicrobial preservation in ophthalmic solutions was selected in preference to the USP test.

The official $E P$ 'Test for the efficacy of antimicrobial preservation' is usually applied to preserved eyedrops to establish preservative efficacy, ${ }^{16}$ but to our knowledge, this is the first time it is being applied to unpreserved eyedrops to establish inherent antimicrobial efficacy.

Table 1 European Pharmacopoeia criteria

\begin{tabular}{llllllll}
\hline & \multicolumn{7}{c}{ Log reduction } \\
\cline { 3 - 7 } Organism & Criterion & $6 h$ & $24 h$ & $7 d$ & $14 d$ & $28 d$ \\
\hline Calbicans and $A$ niger & A & & & 2 & & NI \\
& B & A & & & & 1 & NI \\
& B & 2 & 3 & & & NR \\
& B & & 1 & 3 & & NI \\
\hline
\end{tabular}

$\mathrm{NI}=$ no increase, $\mathrm{NR}=$ no recovery
TEST METHOD

A total of 21 commonly used unpreserved eyedrops ${ }^{15}$ were tested for inherent antimicrobial efficacy in accordance with the test described in the $E P^{16}$ using the test organisms Pseudomonas aeruginosa (NCIMB 8626), Staphylococcus aureus (NCTC 10788), Candida albicans (NCPF 3179), and Aspergillus niger (IMI 149007). A series of containers of the product to be examined were each inoculated with a suspension of one of the test organisms to give an inoculum of $10^{5}$ to $10^{6}$ micro-organisms per $\mathrm{ml}$.

The $E P^{16}$ stipulates that during the test for the efficacy of antimicrobial preservation, the inoculated product is maintained at $20^{\circ} \mathrm{C}-$ $25^{\circ} \mathrm{C}$ and protected from light. In order to prove our recommendation that in use unpreserved eyedrops should be stored in a refrigerator at $2^{\circ} \mathrm{C}-8^{\circ} \mathrm{C}$, an initial comparison was made between inoculated eyedrops stored at $20^{\circ} \mathrm{C}-25^{\circ} \mathrm{C}$ (as stipulated in the $E P$ ) and inoculated eyedrops refrigerated at $2^{\circ} \mathrm{C}-8^{\circ} \mathrm{C}$. Unpreserved atropine and chloramphenicol were the two eyedrops used in this part of the study. All subsequent preservative efficacy tests were carried out at $2^{\circ} \mathrm{C}-8^{\circ} \mathrm{C}$ to reflect our requirement that in use unpreserved eyedrops should be refrigerated.

One $\mathrm{ml}$ aliquots of the inoculated product were removed at $0,6,24$, and 48 hours and 7 , 14, and 28 days. Each was added to $9 \mathrm{ml}$ of $0.1 \%$ peptone water containing polysorbate 80 $1 \%$, lecithin $0.5 \%$, Triton X100 $1 \%$, and sodium thiosulphate $1 \%$ as preservative inactivity agents. The control preparations were similarly sampled at 0 hours to determine the viable counts of the cultures used and to confirm the suitability of the media used for their growth.

Further dilutions were made as necessary. One $\mathrm{ml}$ aliquots of all dilutions were incorporated in duplicate pour plates and incubated at $30^{\circ} \mathrm{C}-35^{\circ} \mathrm{C}$ for 3 days for the bacteria, and at $20^{\circ} \mathrm{C}-25^{\circ} \mathrm{C}$ for 5 days for the yeasts and moulds. After incubation, the number of colonies on each plate were counted and taking the dilution factor into account, the number of colony forming units (cfu) per ml of product were calculated.

Each of the eyedrops was diluted 10-fold, 100 -fold, 1000-fold, and 10000 -fold, and $1 \mathrm{ml}$ of each dilution was added to four out of a series of five petri dishes containing 100 viable organisms of one of the test organisms. The fifth petri dish acted as a control. Molten agar was added to each of the plates which were then incubated as described above and examined for growth. This procedure was repeated with each of the four test organisms to establish the validity of the recovery counts.

Unpreserved oily eyedrops (for example, clotrimazole $1 \%$ in arachis oil eyedrops) were not tested, since micro-organisms do not usually grow in non-aqueous media. Also, in order to test such preparations, the method of the $E P$ or USP is not considered suitable, since the inoculum is introduced as an aqueous suspension, thereby altering the microbiological aspects of the test system considerably. ${ }^{17}$ 
Table 2 Comparison of unpreserved atropine 1\% eyedrops stored in the refrigerator with eyedrops stored at room temperature

\begin{tabular}{lllllll}
\hline \multirow{7}{*}{ Organism } & \multicolumn{9}{l}{ Log reduction } \\
\cline { 3 - 7 } & Temperature $\left({ }^{\circ} C\right)$ & $6 h$ & $24 h$ & $7 d$ & $14 d$ & $28 d$ \\
\hline C albicans & $2-8$ & 0.44 & 1.00 & 1.81 & 3.40 & NR \\
& 25 & 0.53 & 0.78 & 1.08 & 1.23 & 4.96 \\
A niger & $2-8$ & 0.69 & 1.05 & 2.51 & NR & NR \\
& 25 & 0.66 & 0.76 & 3.52 & 3.32 & 3.29 \\
Paeruginosa & $2-8$ & NR & NR & NR & NR & NR \\
\multirow{2}{*}{ S aureus } & 25 & 2.54 & 4.51 & NR & NR & NR \\
& $2-8$ & 0.63 & 0.88 & NR & NR & NR \\
& 25 & 0.44 & 0.75 & NR & NR & NR \\
\hline
\end{tabular}

Table 3 Comparison of unpreserved chloramphenicol $0.5 \%$ eyedrops stored in the refrigerator with eyedrops stored at room temperature

\begin{tabular}{llllllll}
\hline \multicolumn{7}{c}{ Log reduction } \\
\cline { 3 - 7 } Organism & Temperature & $\left({ }^{\circ}\right.$ C) & $6 h$ & $24 h$ & $7 d$ & $14 d$ & $28 d$ \\
\hline C albicans & $2-8$ & 0.51 & 0.82 & 2.73 & NR & NR \\
& 25 & 0.25 & 0.64 & 1.20 & 2.60 & NR \\
A niger & $2-8$ & 0.81 & 1.93 & 2.17 & 2.98 & 3.22 \\
& 25 & 1.12 & 1.34 & 3.49 & 3.31 & 3.31 \\
Paeruginosa & $2-8$ & 1.00 & 1.43 & NR & NR & NR \\
& 25 & 0.57 & 0.88 & NR & NR & NR \\
Saureus & $2-8$ & 0.83 & 0.95 & 3.05 & NR & NR \\
& 25 & 0.49 & 0.69 & NR & NR & NR \\
\hline
\end{tabular}

Table 4 Results of unpreserved eyedrops tested at $2-8^{\circ} \mathrm{C}$. Log reductions or increases in count are given as actual whole number values recorded, except where they pass the specified $E P$ standard, criterion $A$ or criterion $B$

\begin{tabular}{|c|c|c|c|c|}
\hline & C albicans & $A$ niger & $P$ aeruginosa & $S$ aureus \\
\hline Unpreserved eyedrops & $17 d 14 d 28 d$ & $7 d 14 d 28 d$ & $6 h 24 h 7 d 28 d$ & $6 h 24 h 7 d 28 d$ \\
\hline Homatropine $1 \%$ & Pass A & Pass A & Pass A & Pass B \\
\hline Phenylephrine $10 \%$ & Pass B & Pass A & Pass A & Pass B \\
\hline Chloramphenicol $0.5 \%$ & Pass A & Pass A & Pass B & $<1<1>3 \mathrm{NR}$ \\
\hline Gentamicin $1.5 \%$ & $>1<1<1$ & Pass A & Pass A & Pass A \\
\hline Gentamicin $0.3 \%$ & $>1<1<1$ & Pass A & Pass A & Pass A \\
\hline Cyclopentolate $1 \%$ & Pass B & Pass B & Pass A & $<1<1$ NR NR \\
\hline Atropine $1 \%$ & Pass B & Pass A & Pass A & $<1<1$ NR NR \\
\hline Pilocarpine $0.5 \%$ & Pass B & Pass B & Pass A & $<1<1$ NR NR \\
\hline Pilocarpine $1 \%$ to $4 \%$ & $>1<1<1$ & $>1<1<1$ & Pass A & $<1<1 \mathrm{NR}$ NR \\
\hline Cefuroxime $5 \%$ & Pass B & $>1 \mathrm{NI}<1$ & Pass B & NR NR NR >1 \\
\hline Acetylcysteine 5\% & Pass A & Pass B & Pass A & $<1<1>1>2$ \\
\hline Citrate $6.5 \%$ & Pass B & Pass B & Pass A & $<1<1>1>1$ \\
\hline Hypromellose $0.3 \%$ & Pass A & Pass A & Pass A & $<1<1>1<1$ \\
\hline Potassium ascorbate & Pass B & Pass B & Pass A & $<1<1>1$ NR \\
\hline Sodium chloride $5 \%$ & Pass A & Pass B & Pass A & $<1<1>1 \mathrm{NR}$ \\
\hline Dexamethasone $0.1 \%$ & $<1<1<1$ & Pass B & Pass B & $<1<13 \mathrm{NR}$ \\
\hline Sodium chloride $0.9 \%$ & Pass B & Pass B & $>1>1>2>3$ & $<1<1>1>3$ \\
\hline Povidone $0.5 \%$ & Pass B & Pass B & $>1>1>2>4$ & $<1<1>1>1$ \\
\hline Prednisolone $0.01 \%$ & Pass B & Pass B & $>2>2>2<2$ & $<1<1>1>1$ \\
\hline Sodium bicarbonate $2 \%$ & Pass A & $>1<1>1$ & $>2>3>4>3$ & $<1<1<1<1$ \\
\hline Sodium cromoglycate $2 \%$ & Pass B & Pass B & $<1<1>1<1$ & $<1<1>1<1$ \\
\hline Penicillin $0.3 \%$ & $<1<1<1$ & Pass B & $<1<1>2>3$ & Pass A \\
\hline
\end{tabular}

INTERPRETATION OF MICROBIAL COUNTS

The $E P$ criteria for evaluation of antimicrobial activity for ophthalmic preparations, $E P(A)$ and $E P(B)$, are given in terms of the log reduction in the number of viable microorganisms against the value obtained for the inoculum, and are summarised in Table 1. The A criterion expresses the recommended efficacy to be achieved. In justified cases where the A criterion cannot be attained, the $B$ criterion must be satisfied.

By interpreting the results for the various unpreserved eyedrops tested, it is possible to give general recommendations for an in use storage life for the eyedrops tested.

Colony counting is not a precision method, and considering that the criteria are given in integer logarithmic reduction values, a half $\log$ reduction value was taken to specify the requirement of 'no increase' (NI)..$^{17}$

\section{Results}

Atropine $1 \%$ eyedrops without preservative stored at either $20^{\circ} \mathrm{C}-25^{\circ} \mathrm{C}$ or $2^{\circ} \mathrm{C}-8^{\circ} \mathrm{C}$ failed to meet the $E P(\mathrm{~B})$ specification with respect to $S$ aureus, as shown in Table 2.

However, at $2^{\circ} \mathrm{C}-8^{\circ} \mathrm{C}$, there is a greater reduction in $C$ albicans and $P$ aeruginosa, and the drops only just failed the $E P$ (B) criterion in that $S$ aureus in not reduced by $1 \log$ in 24 hours.

Table 3 shows that chloramphenicol $0.5 \%$ unpreserved eyedrops stored at room temperature failed the $E P$ (A) criterion for $P$ aeruginosa, $S$ aureus, and $C$ albicans, as well as failing the $E P$ (B) criterion for $P$ aeruginosa and $S$ aureus. However, at $2^{\circ} \mathrm{C}-8^{\circ} \mathrm{C}$, they satisfy $E P$ (A) for $C$ albicans and $A$ niger, meet the $E P$ (B) for $P$ aeruginosa, while only just failing the $E P$ (B) criterion in that $S$ aureus is not reduced by $1 \log$ in 24 hours.

Further efficacy testing was carried out only at $2^{\circ} \mathrm{C}-8^{\circ} \mathrm{C}$, and results are reported in Table 4 in decreasing range of efficacy, with the first results being for unpreserved eyedrops showing the greatest degree of inherent antimicrobial activity.

\section{Discussion}

The results show a lower rate of replication of organisms in refrigerated samples, and this is reflected in the greater log reductions achieved. This confirms the importance of refrigeration of unpreserved aqueous eyedrops.

The results show that many unpreserved eyedrops have their own inherent antimicrobial activity and many even achieve criterion $E P$ (A) against some micro-organisms. In ophthalmology, it is justifiable to accept the lower EP (B) criterion in cases including intolerance to preservatives as a result of allergy or hypersensitivity, ${ }^{18}$ use in patients with a compromised epithelium, ${ }^{19}$ or use in patients requiring intensive or chronic antiglaucoma therapy. ${ }^{20-29}$

The inability of unpreserved chloramphenicol $0.5 \%$ eyedrops to achieve the $E P$ (B) standard against $S$ aureus is surprising, as clinically this is usually the treatment of choice for $S$ aureus infections! This inconsistency between in vitro and in vivo testing calls into question the validity of in vitro challenge testing, although the discrepancy could be explained by the bacteriostatic nature of chloramphenicol.

Several unpreserved eyedrops, including gentamicin, cyclopentolate, atropine, pilocarpine, and cefuroxime, show a satisfactory reduction in the level of micro-organisms within 7 days, but cefuroxime, gentamicin, and some strengths of pilocarpine showed an increase thereafter, confirming that a 7 day life should not be exceeded as recommended by some authors. ${ }^{30}$

Eyedrops such as acetylcysteine, hypromellose, and sodium chloride $0.9 \%$ have less inherent antimicrobial activity, but they are normally used in intact eyes where there is less risk of ophthalmic infection from contaminated eyedrops than in damaged eyes. Eye- 
drops such as citrate $6.5 \%$ and potassium ascorbate are used in the treatment of chemical burns, where the use of topical antibiotics is mandatory.

Simple saline solutions and eyedrops containing phosphate buffers (for example, prednisolone) appear to have little inherent antimicrobial activity. Conversely, eyedrops which contain disodium edetate have an increased ability to reduce the level of $P$ aeruginosa and therefore consideration must be given to the overall formulation of the products as well as the active ingredients.

Most of the eyedrops tested had least inherent antimicrobial action against $S$ aureus, and this problem is also encountered with products which are preserved. ${ }^{11}{ }^{31}$ Additionally, plasmid resistance has been shown to inactivate certain preservatives, notably mercurials, ${ }^{32}$ so the inclusion of preservatives may not always resolve this dilemma.

To date there is no unequivocal clinical evidence to suggest that eyedrops formulated without a preservative are more likely to result in ocular infections than eyedrops formulated with a preservative. Our experience at Moorfields Eye Hospital over 19 years with the use and production of in excess of 1.3 million bottles of unpreserved eyedrops has shown the 7 day in use, refrigerated, storage life to be a safe and appropriate figure for normal domiciliary use.

\section{Recommendations}

Unpreserved eyedrops should be stored at $2^{\circ} \mathrm{C}-8^{\circ} \mathrm{C}$ after opening to reduce the rate of replication of any microbial contaminants. In general, a 7 day in use storage life is confirmed for eyedrops containing alkaloids or antibiotics once opened by patients in a domiciliary situation.

When deciding upon an in use storage life, consideration should be given to a number of factors, including whether the eyedrops are being used in intact or damaged eyes, whether they are being administered in conjunction with antibiotic therapy, or whether they are being used in a hospital or domiciliary setting. Even in a hospital setting, the risk factors in an acute hospital may be different from those in a long stay hospital. The onus remains on medical practitioners to ensure that unpreserved multidose eyedrops are only used in appropriate circumstances to ensure safe and effective therapy.

We would like to thank Alan Davison for helpful comments and suggestions, and Safepharm Laboratories for assisting with the microbiological tests. This study was sponsored by a Moorfields locally organised research grant.

1 British Pharmacopoeia. London: HMSO, 1993:736.
2 Barkman R, Germanis M, Karpe G, Malmborg S. Preservatives in eyedrops. Acta Ophthalmol 1969;47:461-75.

3 Wilson WS, Duncan AJ, Jay JL. Effect of benzalkonium chloride on the stability of the precorneal tear film in rabbit chloride on the stability of the precorneal tear

4 Tripathi BJ, Tripathi RC. Cytotoxic effects of benzalkonium Tripathi BJ, Tripathi RC. Cytotoxic effects of benzalkonium
chloride and chlorbutanol on human corneal epithelial cells in vitro. Lens Eye Toxic Res 1989;6:395-403.

5 Hertel F, Pfeiffer N. Einzeldosisapplikationen in der Glaukomtherapie. Vergleich der Kosten mit Mehrdosis. Ophthalmologe 1994;91:602-5.

6 British National Formulary. No 28. Control of microbial contamination. London: The Pharmaceutical Press, Sept 1994:399.

7 British Pharmaceutical Codex. London: The Pharmaceutical Press, 1973:685-7.

8 Health Service Circular. Preservation of sterility in ophthalmic preparations in hospitals. London: DHSS, HSC(IS) 122, March 1975 .

9 United States Pharmacopeia, XXIII. Rockville, MD: United United States Pharmacopeia, XXIII. Rockville, MD:
States Pharmacopeial Convention Inc, 1995:1681.

10 Medicines Act Information Leaflet No 45. London: Medicines Control Agency, Department of Health, November 1985

11 Davison AL, Hooper WL, Spooner DF, Farwell JA, Baird R. The validity of the criteria of pharmacopoeial preservative efficacy tests-a pilot study. Pharm $\mathcal{f}$ 1991;246:555-7.

12 Guest HE, Hanlon GW, Livingstone DJ. Microbial evaluation of preservative-free eyedrops in multiple application containers. Pharm $\mathcal{F}$ 1990;245(Suppl 29):R17.

13 Livingstone DV, Guest HE. Keep patients' eyes free of infection. Hospital Pharmacy Practice 1994;4:301-3.

14 Ford JL, Brown MW, Hunt PB. A note on the contamination of eyedrops following use by hospital out-patients. $f$ tion of eyedrops following use by

15 Moorfields Eye Hospital Formulary. London: Pharmacy Department, Moorfields Eye Hospital, 1992.

16 European Pharmacopoeia. 2nd ed. VIII 14. Efficacy of antimicrobial preservation. France: Maisonneuve SA, 1994.

17 Anon. Pharmeuropa 1993;5:355-358.

18 Kruyswijk MR, Polak BC. Contactallergie na toepassing van oogdruppels en oogzalven. Ned Tijdschr Geneeskd 1980;124: 1449-52.

19 Lemp MA, Zimmerman LE. Toxic endothelial degeneration in ocular surface disease treated with topical medications containing benzalkonium chloride. Am $\mathcal{f}$ Ophthalmol 1988; 105:670-3.

20 Van Horn DL, Edelhauser HF, Prodanovich G, Eiferman R, Pederson HF. Effect of the ophthalmic preservative Pederson HF. Effect of the ophthalmic preservative thiomerosal on rabbit and human corneal

21 Collin HB. Ultrastructural changes to corneal stromal cells due to ophthalmic preservatives. Acta Ophthalmol 1986;64: $72-8$.

22 Collin HB, Grabsch BE. The effect of ophthalmic preservatives on the healing rate of the rabbit corneal epithelium after keratectomy. Am $\mathcal{F}$ Optom Physiol Opt 1982;59:21522.

23 Burstein NL. Corneal cytotoxicity of topically applied drugs, vehicles and preservatives. Surv Ophthalmol 1980; 25:15-30.

24 Laflamme MY, Swieca R. A comparative study of two preservative-free tear substitutes in the management of preservative-free tear substitutes in the manage
severe dry eye. Can $f$ Ophthalmol 1988;23:174-6.

25 Seal D, Ficker L, Wright P, Andrews V. The case against thiomersal. Lancet 1991;338:315-6.

26 Gobbels M, Spitznas M. Influence of artificial tears on corneal epithelium in dry-eye syndrome. Graefes Arch Clin Exp Ophthalmol 1989;227:139-41.

27 Mietz H, Niesen U, Krieglstein GK. The effect of preservatives and antiglaucomatous medication on the histopathology of the conjunctiva. Graefes Arch Clin Exp Ophthalmol 1994;232:561-5.

28 Caffery BE, Josephson JE. Is there a better 'comfort drop'? 7 Am Optom Assoc 1990;61:178-82.

29 Marquardt R, Schubert T. Beeinflussung der Tranenfilmaufreisszeit (BUT) durch Betablocker-Augentropfen ohne Konservierungsstoffe. Klin Monatsbl Augenheilkd 1991;199: 75-8.

30 Conrads H, Winterhoff D, Strotmann U. Wert und Unwert von Konservierungsmitteln in Augentropfen. Praxisumfragen und experimentelle Untersuchungen zur Forderung des DAB 8/78. Klin Monatsbl Augenheilkd 1984;184:233-7.

31 Longfield RN, Smith LP, Longfield JN, Coberly J, Cruess D. Multiple-dose vials: persistence of bacterial contaminants and infection control implications. Infection Control 1985;6:194-9.

32 Pinney RJ. Survival of plasmid-containing strains of $E$ coli, $P$ aeruginosa and $S$ aureus in phenylmercuric nitrate and thiomersal. $\mathcal{f}$ Pharm Pharmacol 1978;30:228-32. 